\title{
Color and Local Maximum Edge Patterns Histogram for Content Based Image Retrieval
}

\author{
K. Prasanthi Jasmine ${ }^{1}$, P. Rajesh Kumar ${ }^{2}$ \\ ${ }^{1}$ Research Scholar, ${ }^{2}$ Ass sociate professor; Department of Electronics and Communication Engineering, Andhra \\ University, Visakhapatnam, Andhra Pradesh, India \\ jasminekprasanthi@gmail.com, 2rajeshauce@gmail.com
}

\begin{abstract}
In this paper, HSV color local maximum edge binary patterns (LMEBP) histogram and LMEBP joint histogram are integrated for content based image retrieval (CBIR). The local HSV region of image is represented by LMEBP, which are evaluated by taking into consideration the magnitude of local difference between the center pixel and its neighbors. This LMEBP differs from the existing LBP in a manner that it extracts the information based on distribution of edges in an image. Further the joint histogram is constructed between uniform two rotational invariant first three LMEBP patterns. The color feature is extracted by calculating the histogram on Hue (H), Saturation (S) and LMEBP histogram on Value (V) spaces. The feature vector of the system is constructed by integrating HSV LMEBP histograms and LMEBP joint histograms. The experimentation has been carried out for proving the worth of our algorithm. It is further mentioned that the databases considered for experiment are Corel-1K and Corel-5K. The results after being investigated show a significant improvement in terms of their evaluation measures as compared to previously available spatial and transform domain methods on their respective databases.
\end{abstract}

Index Terms - Color; Texture; Feature Extraction; Local Binary Patterns; Local Maximum Edge Binary Patterns; Image Retrieval

\section{INTRODUCTION}

The Digital media is evolving so digital data archives in scientific, industrial, medical, environmental, educational, entertainment, web image searching and other applications. For accessing digital images in these applications it is required to have an efficient algorithm. Before 1990 text based image retrieval was being used for this purpose. To reduce the amount of labor for image annotation and different interpretation of image by different persons, in early 1990 CBIR came into picture [1]. Still there remain some challenging problems that attract the researchers' interest towards CBIR. Generally in CBIR, visual features related to color, shape, texture, and spatial layout are extracted in the form of color histogram [2], color coherence vector [3], color correlogram (CC) [4], Gabor transform [5] etc. These are arranged as multidimensional feature vectors and construct the feature database. For similarity/distance measurement many methods have been developed like Euclidean distance, city-block distance etc. Selection of feature descriptors and similarity/distance measures affect retrieval performances of an image retrieval system significantly. Moghaddam et al. proposed the Gabor wavelet correlogram (GWC) for CBIR [6, 7]. Ahmadian et al. used the wavelet transform for texture classification [8]. Moghaddam et al. introduced new algorithm called wavelet correlogram (WC) [9]. Saadatmand et al. [10, 11] improved the performance of WC algorithm by optimizing the quantization thresholds using genetic algorith m (GA). Birgale et al. [12] and Subrah manyam et al. [13] combined the color (color histogram) and texture (wavelet transform) features for CBIR. Subrah manyam et al. proposed correlogram algorith $m$ for image retrieval using wavelets and rotated wavelets (WC+RWC) [14].

In development of local feature descriptor, scale invariant feature transform (SIFT) feature is proposed by Lowe et al. [15] in 2004. SIFT feature descriptor is invariant to scale, orientation and partially invariant to affine distortion and illumination changes. In SIFT the locations of extrema in difference of Gaussian (DoG) correspond to most stable feature with respect to scale variance, and are identified as interest feature points or keypoints. For each keypoint sequel HOG descriptor are calculated. Thus HOG feature is assumed to be orig inated from SIFT descriptor. HOG feature descriptor is introduced by Dalal et al. [16] in 2005 for solving the problem of pedestrian detection in static images. Ojala et al. proposed the local binary patterns (LBP) for texture description [17] and these LBPs are converted to rotational invariant for texture classification [18]. Pietikainen et al. proposed the rotational invariant texture classification using feature distributions [19]. Ahonen et al. [20] and Zhao et al [21] used the LBP operator facial expression analysis and recognition. Heikkila et al. proposed the background modeling and detection by using LBP [22]. Huang et al. proposed the extended LBP for shape localization [23]. Heikkila et al. used the LBP for interest region description [24]. Li et al. used the combination of Gabor filter and LBP for texture segmentation [25]. Zhang et al. proposed the local derivative pattern for face recognition [26]. They have considered LBP as a nondirectional first order local pattern, which are the binary results of the first-order derivative in images. Abdullah et al. [27] proposed fixed partitioning and salient points schemes for dividing an image into patches, in combination with low-level MPEG-7 visual descriptors to represent the patches with particular patterns. A clustering technique is applied to construct a compact representation by grouping similar 
patterns into a cluster codebook. The codebook will then be used to encode the patterns into visual keywords. In order to obtain high-level information about the relational context of an image, a correlogram is constructed from the spatial relations between visual key word indices in an image. For classifying images k-nearest neighbors (k-NN) and a support vector machine (SVM) algorithm are used and compared. Jhanwar et al. [28] have proposed the motif co-occurrence matrix (MCM) for content based image retrieval. The MCM is derived from the motif transformed image which is calculated by dividing the whole image into non-overlapping $2 \times 2$ pixel patterns. They also proposed the color MCM which is calculated by applying MCM on individual red (R), green (G), and blue (B) color planes. C H Lin et al. [29] combined the color feature, k-mean color histogram (CHKM) and texture features, motif co-occurrence matrix (MCM) and difference between the pixels of a scan pattern (DBPSP). MCM is the conventional pattern co-occurrence matrix that calculates the probability of the occurrence of same motif between each motif and its adjacent ones in each motif transformed image, and this probability is considered as the attribute of the image. According to the sequence of motifs of scan patterns, DBPSP calculates the difference between pixels and converts it into the probability of occurrence on the entire image. Each pixel color in an image is then replaced by one color in the common color palette that is most similar to color so as to classify all pixels in image into $\mathrm{k}$-cluster, called the CHKM feature. Subrahmanyam et al. [30] have proposed the local maximum edge binary patterns (LMEBP) for image retrieval and object tracking applications. Their method extracts the features by taking into consideration the magnitude of local difference between the center pixel and its neighbors.

To improve the retrieval performance in terms of retrieval accuracy, in this paper, we combine the color (HSV LMEBP histogram) and texture (LMEBP joint histogram) features. The experiments have been carried out on Corel- $1 \mathrm{~K}$ and Corel-5K databases for proving the worth of our algorithm. The results after investigation show a significant improvement in terms of their evaluation measures as compared to other existing color $\&$ texture and spatial and transform domain features.

The organization of the paper as follows: In section I, a brief review of image retrieval and related work is given. Section II, III and IV presents a concise review of local binary patterns, local maximum edge binary patterns and HSV histogram calculation respectively. Section V, presents the proposed system framework and similarity measure. Experimental results and discussions are given in section VI. Based on above work conclusions are derived in section VII.

\section{LOCAL BINARY PATTERNS}

The LBP operator was introduced by Ojala et al. [17] for texture classification. Success in terms of speed (no need to tune any parameters) and performance is reported in many research areas such as texture classification [1719], face recognition [24-26], object tracking [30], biomedical image retrieval [31] and finger print recognition.

Given a center pixel in the $3 \times 3$ pattern, LBP value is computed by comparing its gray scale value with its neighborhoods based on (1) and (2):

$$
\begin{aligned}
& L B P_{P, R}=\sum_{i=1}^{P} 2^{(i-1)} \times f\left(I\left(g_{i}\right)-I\left(g_{c}\right)\right) \\
& f(x)= \begin{cases}1 & x \geq 0 \\
0 & \text { else }\end{cases}
\end{aligned}
$$

where $I\left(g_{c}\right)$ denotes the gray value of the center pixel, $I\left(g_{i}\right)$ is the gray value of its neighbors, $P$ stands for the number of neighbors and $R$, the radius of the neighborhood.

Fig. 1 shows an example of obtaining an LBP from a given $3 \times 3$ pattern. The histograms of these patterns extract the distribution of edges in an image.

\begin{tabular}{|c|c|c|}
\hline \multicolumn{3}{|c|}{ Binary Pattern } \\
\hline 1 & 0 & 0 \\
\hline 1 & & 0 \\
\hline 1 & 1 & 1 \\
\hline
\end{tabular}

Example
\begin{tabular}{|c|c|c|}
\hline 6 & 5 & 2 \\
\hline 7 & 6 & 1 \\
\hline 9 & 8 & 7 \\
\hline
\end{tabular}

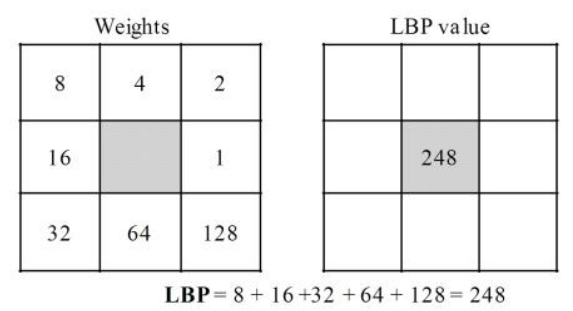

Fig. 1. LBP calculation for $3 \times 3$ pattern

\section{LOCAL MAXIMUM EDGE BINARY PATTERNS}

Subrahmanyam et al. [30] have proposed the local maximum edge binary patterns (LMEBP) for image retrieval and object tracking applications. In proposed LMEBP for a given image the first maximum edge is obtained by the magnitude of local difference between the center pixel and its eight neighbors as shown below:

$$
\begin{aligned}
& I^{\prime}\left(g_{i}\right)=I\left(g_{c}\right)-I\left(g_{i}\right) ; \quad i=1,2, \ldots \ldots, 8 \\
& \left.i_{1}=\underset{i}{\arg (\max }\left(\left|I^{\prime}\left(g_{1}\right)\right|,\left|I^{\prime}\left(g_{2}\right)\right|, \ldots \ldots .,\left|I^{\prime}\left(g_{8}\right)\right|\right)\right)
\end{aligned}
$$

where, $\max (x)$ calculates the maximum value in an array ' $x$ '.

If this edge is positive, assign ' 1 ' to this particular center pixel otherwise ' 0 '. 


$$
\begin{aligned}
& I^{\text {new }}\left(g_{c}\right)=f\left(I^{\prime}\left(g_{i_{1}}\right)\right) \\
& f(x)= \begin{cases}1 & x \geq 0 \\
0 & \text { else }\end{cases}
\end{aligned}
$$

The LMEBP is defined as:

$$
\operatorname{LMEBP}\left(I\left(g_{c}\right)\right)=\left\{\begin{array}{c}
I^{\text {new }}\left(g_{1}\right) ; I^{\text {new }}\left(g_{2}\right) ; \\
\ldots \ldots \ldots I^{\text {new }}\left(g_{8}\right)
\end{array}\right\}
$$

The uniform pattern refers to the uniform appearance pattern which has limited discontinuities in the circular binary presentation. In this paper, the pattern which has less than or equal to two discontinuities in the circular binary presentation is considered as the uniform pattern and remaining patterns considered as non-uniform patterns.

Fig. 2 shows all uniform patters for $P=8$. The distinct values for given query image is $P(P-1)+3$ by using uniform patterns. But these features are not rotational invariant.

The rotational invariant patterns $\left(L M E B P_{P, R}^{r i u 2}\right)$ can be constructed by adding all eight patterns in the each row of Fig. 2 as shown in Fig. 3. The distinct values for a given query image is $P+2$ by using rotational invariant patterns $\left(L M E B P_{P, R}^{r i u 2}\right)$. After LMEBP calculation, the joint histogram is constructed between first, second and third LMEBPs for a feature vector generation.

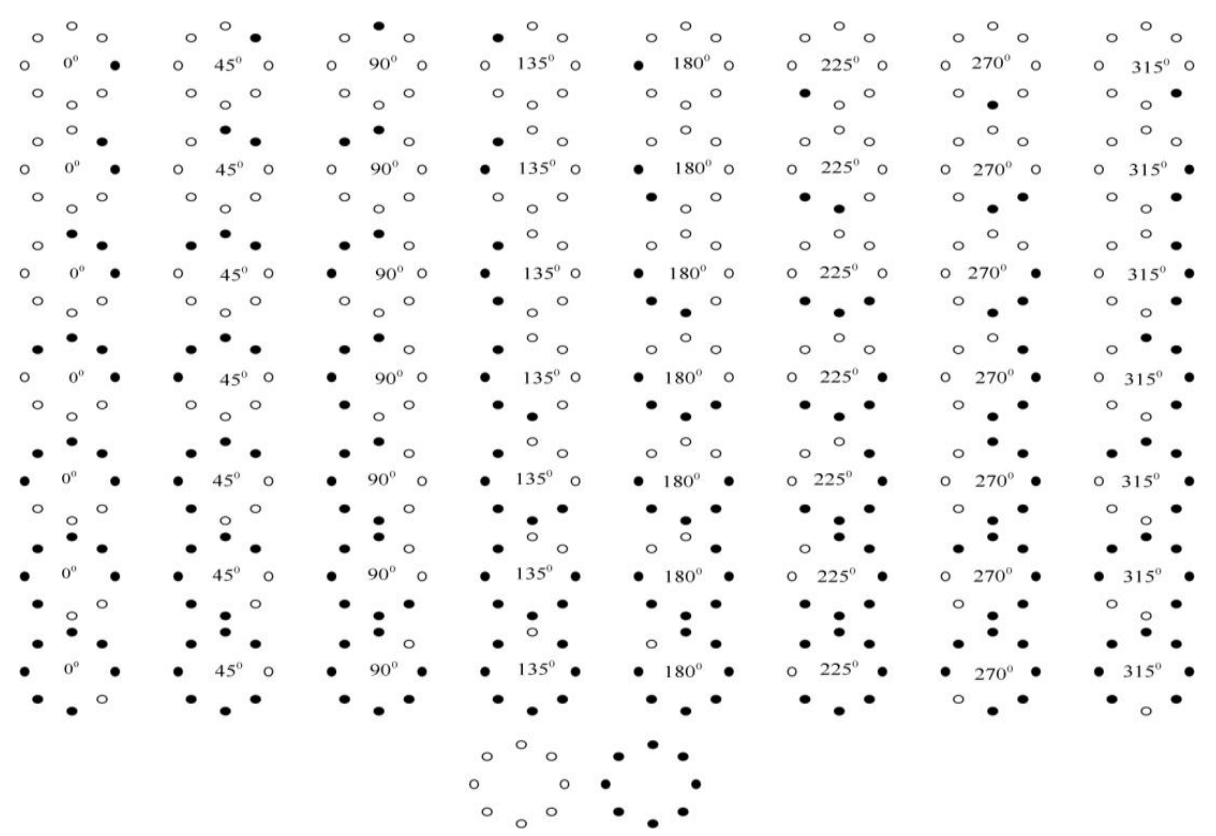

Fig. 2. Uniform patters when $\mathrm{P}=8$. The black and white dots represent the bit values of 1 and 0 in the LMEBP op erator

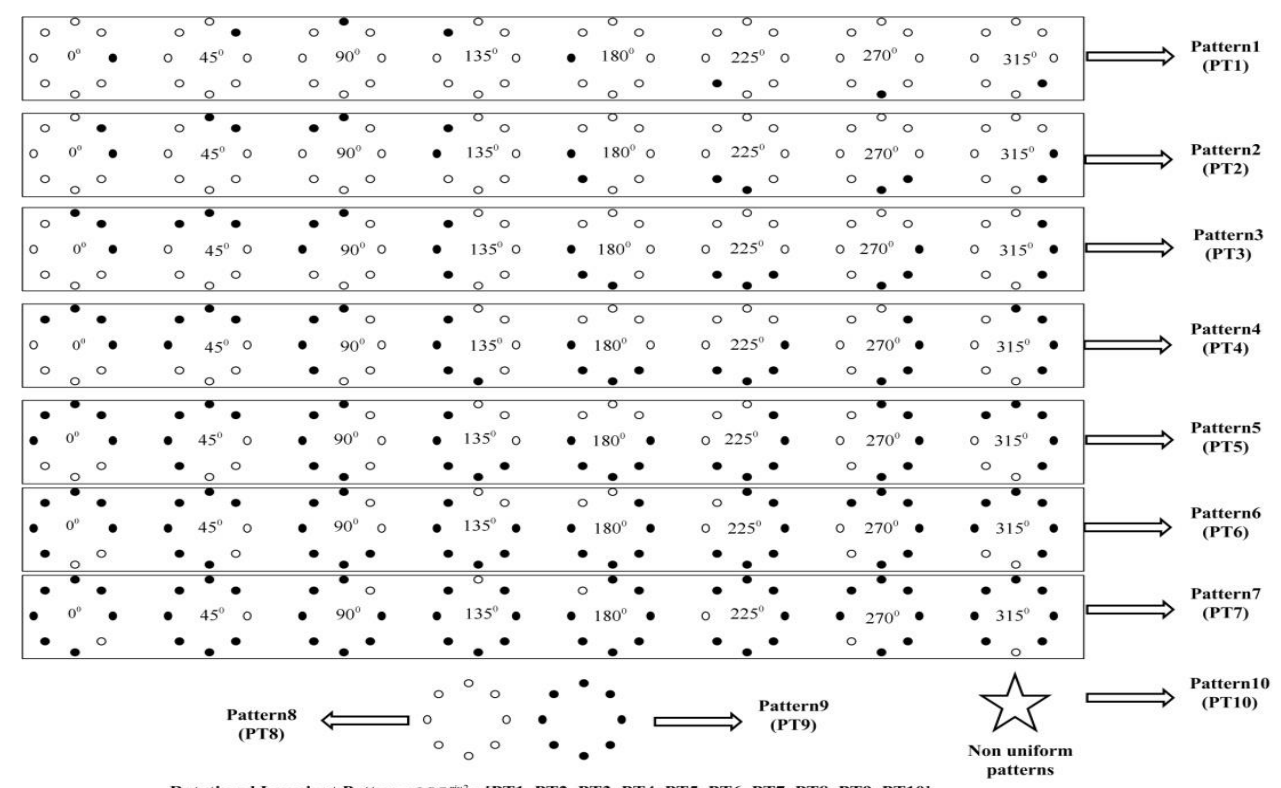

Fig. 3. Rotational variant LMEBP patterns are converted into rotational invariant LMEBP patterns 


\section{HSV COLOR HISTOGRAM}

The color histogram is obtained by counting the number of times each color occurs in the image array. The histogram is invariant to translation and rotation of the image plane, and change only slowly under change of angle of view.

A color histogram $H$ for a given image is defined as a vector

$$
H=\{H[0], H[1], \ldots . ., H[i], \ldots . H[N]\}
$$

where $i$ represent the color in color histogram and $H[\mathrm{i}]$ represent the number of pixels of HSV color $i$ in the image, and $N$ is the number of bins used in color histogram. For comparing the histogram of different sizes, color histogram should be normalized. The normalized color histogram is given as

$$
H^{\prime}=\frac{H}{p}
$$

where $p$ is the total number of pixels in the image.

In this paper, $\mathrm{H}$ and $\mathrm{S}$ color spaces are used for histogram and $\mathrm{V}$ color space is used for the LMEBP histogram for HSV LMEBP histogram feature.

\section{PROPOSED SYSTEM FRAMEWORK}

In this paper, we proposed the new technique by combining joint LMEBP histogram and HSV color histogram for image retrieval. For texture feature, RGB (red, green, and blue) image is converted into gray scale image, then the LMEBP patterns, which are evaluated by taking into consideration of local difference between the pixels. Further, the joint histogram is constructed between first, second and third rotational invariant uniform LMEBP patterns. For color feature, H, S color his tograms and LMEBP on V space are calculated. Finally, feature vector is constructed by concatenating the color and texture features and then these are used for image retrieval. Fig. 4 illustrates the flowchart of proposed systemand algorithm for the same is given below:

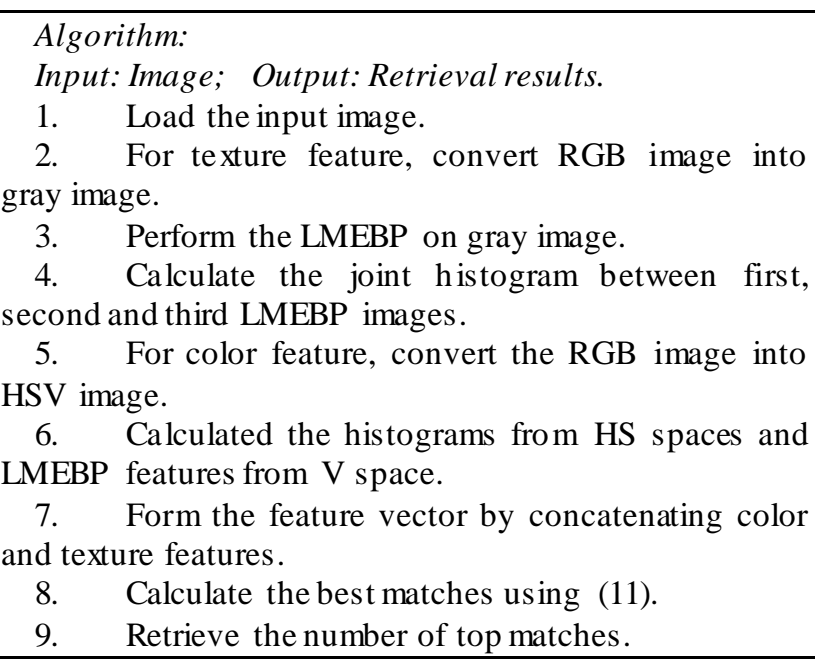

\section{A. Similarity Measurement}

In the presented work four types of similarity distance metric ares used as shown follows.

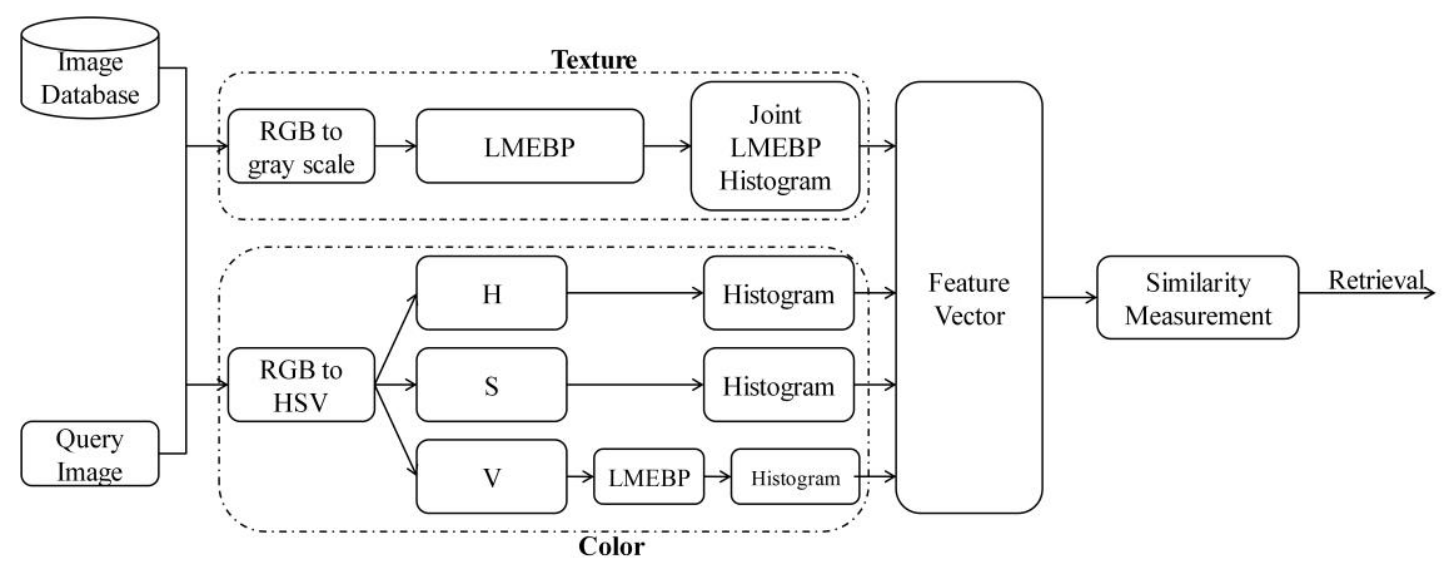

Fig. 4. Flowchart of proposed system

\section{Manhattan or $L_{1}$ or city-block Distance}

This distance function is computationally less expensive than Euclidean distance because only the absolute differences in each feature are considered. This distance is sometimes called the city block distance or $L_{1}$ distance and defined as

$$
D(Q, T)=\sum_{i}\left|f_{i}(Q)-f_{j}(T)\right|
$$

\section{Euclidean or $L_{2}$ Distance}

For $p=2$ in the equation (1.1) give the Euclidean distance and defined as:

$$
D(Q, T)=\left(\sum_{i}\left|f_{i}(Q)-f_{j}(T)\right|^{2}\right)^{1 / 2}
$$

The most expensive operation is the computation of square root.

$D_{1}$ Distance

$$
D(Q, T)=\sum_{i=1}^{L g}\left|\frac{f_{T, i}-f_{Q, i}}{1+f_{T, i}+f_{Q, i}}\right|
$$




$$
D(Q, T)=\sum_{i=1}^{L g} \frac{\left|f_{T, i}-f_{Q, i}\right|}{\left|f_{T, i}+f_{Q, i}\right|}
$$

where $Q$ is query image, $L g$ is feature vector length, $T$ is image in database; $f_{I, i}$ is $i^{\text {th }}$ feature of image $I$ in the database, $f_{Q, i}$ is $i^{\text {th }}$ feature of query image $Q$.

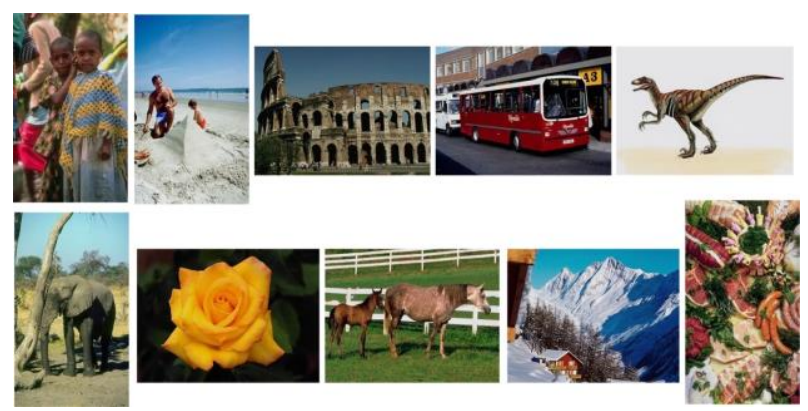

Fig. 5. Some sample images from database Corel-1K (one image per category)

\section{EXPERIMENT AL RESULTS AND DISCUSSIONS}

For the work reported in this paper, retrieval tests are conducted on Corel-1K and Corel-5K and results are presented in the following subsections.

\section{A. Corel-1K Database}

Corel database [35] consists of large nu mber of images of various contents ranging from animals to outdoor sports to natural images. These images have been preclassified into different categories each of size 100 by domain professionals. So me researchers think that Corel database meets all the requirements to evaluate an image retrieval system, due its large size and heterogeneous content. We have collected 1000 images to form database Core $1-1 \mathrm{~K}$. These images are collected from 10 different domains namely Africans, beaches, buildings, buses, dinosaurs, elephants, flowers, horses, mountains and food. Each category has $N_{G}$ (100) images with resolution of either $256 \times 384$ or $384 \times 256$. Fig. 5 shows the sample images of Corel-1K database (one image from each category).

In all experiments, each image in the database is used as the query image. For each query, the system collects $n$ database images $X=\left(x_{1}, x_{2}, \ldots, x_{n}\right)$ with the shortest image matching distance computed using (12). If the retrieved image $x_{i}=1,2, \ldots, n$ belongs to same category as that of the query image then we say the system has appropriately identified the expected image else the system fails to find the expected image.

The performance of the proposed method is measured in terms of average precision, average recall and average retrieval rate (ARR) as shown below:

$$
\begin{aligned}
& \text { Precision }(P)=\frac{\text { No.of Relevant Images Retrieved }}{\text { Total No.of Images Retrieved }} \times 100 \\
& \text { Group Precision }(G P)=\frac{1}{N_{1}} \sum_{i=1}^{N_{1}} P
\end{aligned}
$$

Average Retrieval Precision $(A R P)=\frac{1}{\Gamma_{1}} \sum_{j=1}^{\Gamma_{1}} G P$

$$
\operatorname{Recall}(R)=\frac{\text { Number of relevant images retrieved }}{\text { Total Number of relevant images }}
$$

$$
\operatorname{Group} \operatorname{Recall}(G R)=\frac{1}{N_{1}} \sum_{i=1}^{N_{1}} R
$$

$$
\text { Average Retrieval Rate }(A R R)=\frac{1}{\Gamma_{1}} \sum_{j=1}^{\Gamma_{1}} G R
$$

where $N_{1}$ is number of relevant images and $\Gamma_{1}$ is number of groups.

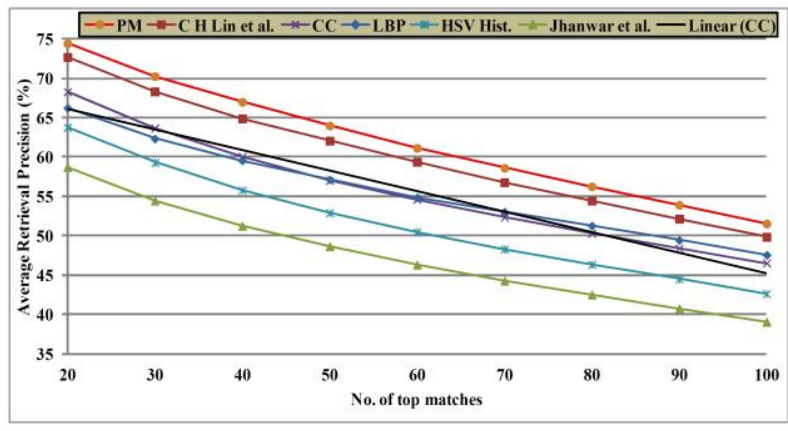

(a)

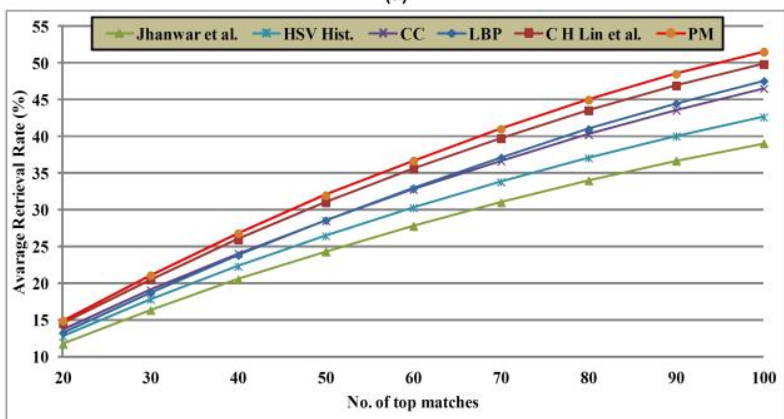

(b)

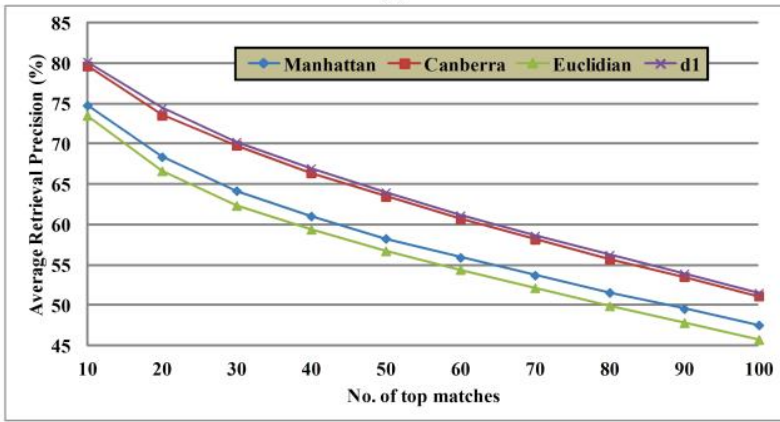

(c)

Fig. 6. Comparison of proposed method with other existing methods in terms: (a)\&(c) average retrieval precision, (b) average retrieval rate on Corel-1 K database.

Table I \& Fig. 6 (a) and Table II \& Fig. 6 (b) summarize the retrieval results of various methods in terms of average retrieval precision and average retrieval rate respectively. Fig. 6 (c) summarize the performance of proposed method with different distance measures in terms of average retrieval rate. 
From the Tables I to II and Fig. 6 the following points can be observed:

1. The average retrieval precision of proposed method (Joint_LMEBP_HSV_Hist) $(75.0 \%$ to $53.5 \%)$ is more as compared to LBP (66.2\% to $47.5 \%)$ and Jhanwar et al. $(58.7 \%$ to $39.0 \%), \mathrm{C} \mathrm{H}$ Lin et al. (72.7\% to $49.8 \%)$, CC $(68.2 \%$ t0 $46.5 \%)$ and HSV Hist. (63.7\% to $42.6 \%)$.

2. The performance of the proposed method with $d_{1}$ distance is more as compared to Canberra, Euclidean and Manhattan distances in terms of average retrieval precision.

From Tables I to II, Fig. 6, and above observations, it is clear that the proposed method is outperforming the other existing techniques in terms of ARR and ARP.

\section{B. Corel-5K Database}

In this experiment, we have used 5000 images to form database of Corel-5K. These images are collected from 50 different domains have $N_{G}$ (100) images. The performance of the proposed method is measured in terms of average precision, average recall, and average retrieval rate (ARR) as shown in (14-19).

Table III illustrates the retrieval results of proposed method and other existing methods on Corel-5K in terms of average precision and recall. Fig. 7 (a) and (b) show the category wise performance of methods in terms of precision and recall on Corel-5K database. The performance of all techniques in terms of average precision and ARR on Corel-5K database can be seen in Fig. 7 (c) and (d) respectively. From Table III and Fig. 7, it is clear that the proposed method shows a significant improvement as compared to other existing methods in terms of their evaluation measures on Corel-5K database. Fig. 8 illustrates the query results of proposed method on Core $1-1 \mathrm{~K}$ and Core $1-5 \mathrm{~K}$ database (top left image is the query image).

Table 1. Results of all techniques in terms of precision on Corel-1K database PM: HSV_LMEBP_Hist+Joint_LMEBP

\begin{tabular}{|c|c|c|c|c|c|c|c|}
\hline \multirow{2}{*}{ Category } & & \multicolumn{7}{|c|}{ Precision $(\boldsymbol{n = 2 0})(\boldsymbol{\%})$} \\
\cline { 2 - 8 } & Jhanwar et al. & C H Lin et al & CC & LBP & LMEBP & HSV Hist. & PM \\
\hline Africans & 53.15 & 68.3 & 80.4 & 56.9 & 57.2 & 66.7 & 80.4 \\
\hline Beaches & 43.85 & 54 & 41.2 & 51.9 & 53.8 & 32.2 & 47.4 \\
\hline Buildings & 48.7 & 56.2 & 55.6 & 61.6 & 63.5 & 56.8 & 79.2 \\
\hline Buses & 82.8 & 88.8 & 76.7 & 95.1 & 96.1 & 83.4 & 92.4 \\
\hline Dinosaurs & 95 & 99.3 & 99 & 98.5 & 100.1 & 99.8 & 99.2 \\
\hline Elephants & 34.85 & 65.8 & 56.2 & 39.9 & 40.1 & 50.6 & 64.3 \\
\hline Flowers & 88.35 & 89.1 & 92.9 & 89.5 & 90.3 & 66.2 & 85.3 \\
\hline Horses & 59.35 & 80.3 & 76.5 & 65.6 & 67.4 & 87.2 & 88.4 \\
\hline Mountains & 30.8 & 52.2 & 33.7 & 37.4 & 39.0 & 24.7 & 33.8 \\
\hline Food & 50.4 & 73.3 & 70.6 & 65.7 & 67.6 & 69.9 & 79.8 \\
\hline TOTAL & $\mathbf{5 8 . 7}$ & $\mathbf{7 2 . 7}$ & $\mathbf{6 8 . 2}$ & $\mathbf{6 6 . 2}$ & $\mathbf{6 7 . 7}$ & $\mathbf{6 3 . 7}$ & $\mathbf{7 5 . 0}$ \\
\hline
\end{tabular}

Table 2. Results of all techniques in terms of recall on Corel-1 $\mathrm{K}$ database PM: HSV_LMEBP_Hist+Joint_LMEBP

\begin{tabular}{|c|c|c|c|c|c|c|c|}
\hline \multirow{2}{*}{ Category } & \multicolumn{5}{|c|}{ Recall $(\boldsymbol{n = 1 0 0})(\boldsymbol{\%})$} \\
\cline { 2 - 8 } & Jhanwar et al. & C H Lin et al & CC & LBP & LMEBP & HSV Hist. & PM \\
\hline Africans & 32.2 & 42.1 & 46.2 & 38.4 & 40.04 & 42.9 & 50.5 \\
\hline Beaches & 29.0 & 32.1 & 25.2 & 35.7 & 37.53 & 19.4 & 28.5 \\
\hline Buildings & 27.7 & 36.5 & 35.0 & 35.9 & 36.18 & 34.9 & 49.5 \\
\hline Buses & 48.6 & 61.7 & 60.9 & 72.2 & 74.08 & 63.7 & 74.8 \\
\hline Dinosaurs & 81.4 & 94.1 & 89.5 & 91.3 & 92.56 & 90.3 & 91.8 \\
\hline Eephants & 21.4 & 33.1 & 34.1 & 26.8 & 26.99 & 29.0 & 36.5 \\
\hline Flowers & 63.5 & 75 & 77.6 & 66.3 & 66.87 & 32.8 & 54.5 \\
\hline Horses & 35.8 & 47.6 & 36.1 & 42.3 & 43.39 & 50.8 & 55.1 \\
\hline Mountains & 21.7 & 27.7 & 21.0 & 26.6 & 28.53 & 14.5 & 22.2 \\
\hline Food & 29.0 & 49 & 39.2 & 40.0 & 41.95 & 47.8 & 51.7 \\
\hline TOTAL & $\mathbf{3 9 . 0}$ & $\mathbf{4 9 . 8}$ & $\mathbf{4 6 . 5}$ & $\mathbf{4 7 . 5}$ & $\mathbf{4 8 . 8}$ & $\mathbf{4 2 . 6}$ & $\mathbf{5 3 . 5}$ \\
\hline
\end{tabular}

Table 3. Results of all methods in terms of precision and recall on Corel-5K and Corel-10K databases PM: Proposed Method; BLK_LBP: Block based LBP [34]

\begin{tabular}{|c|c|c|c|c|c|c|c|c|}
\hline Database & Performance & & \multicolumn{6}{|c|}{ Method } \\
\hline & & CS_LBP [32] & LEPSEG [33] & LEPINV [33] & BLK_LBP [34] & LBP & LMEBP [30] & PM \\
\hline Corel-5K & Precision (\%) & 32.9 & 41.5 & 35.19 & 45.7 & 43.6 & 44.3 & 62.35 \\
\hline
\end{tabular}




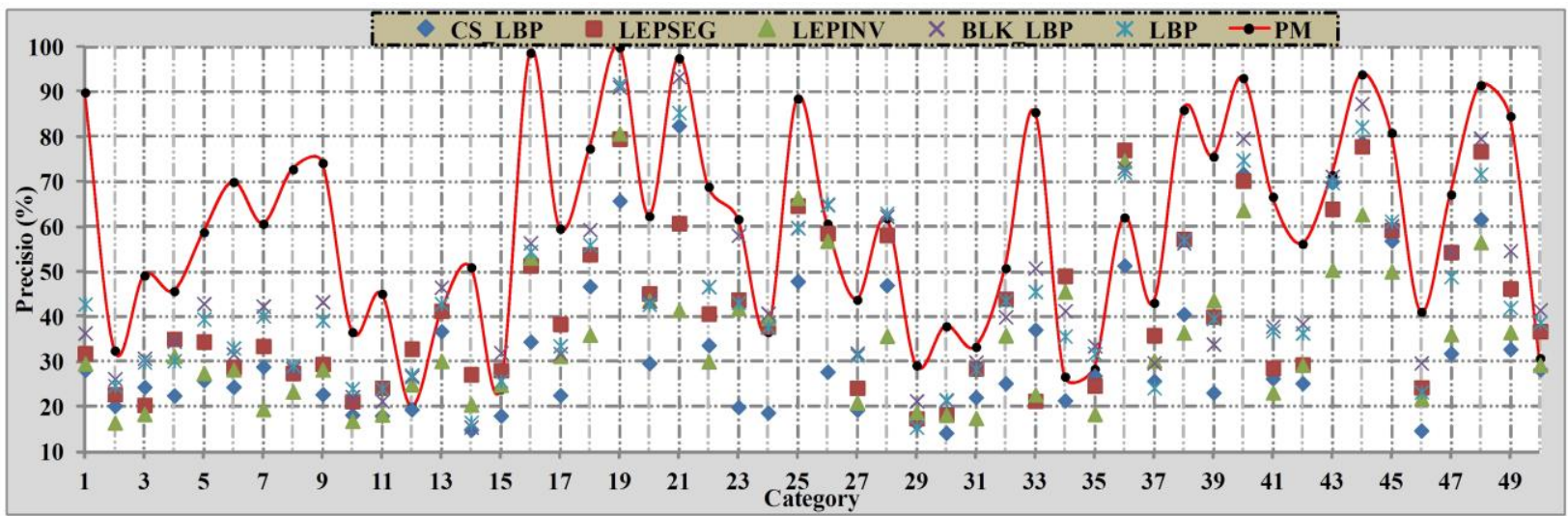

(a)

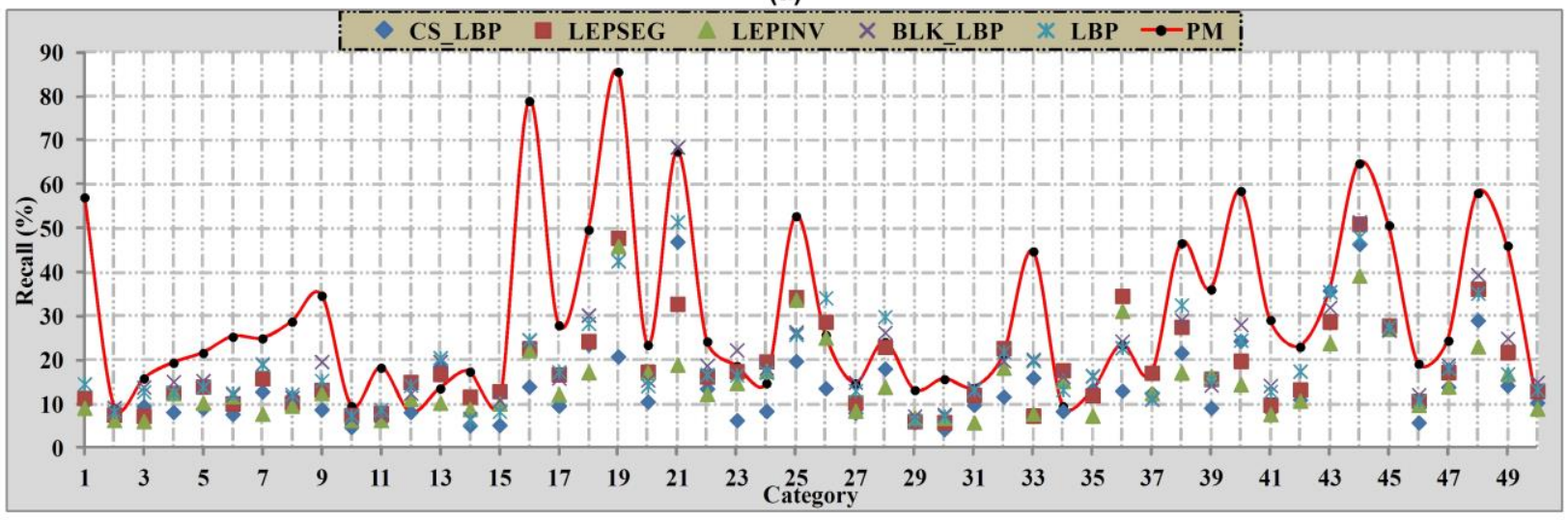

(b)

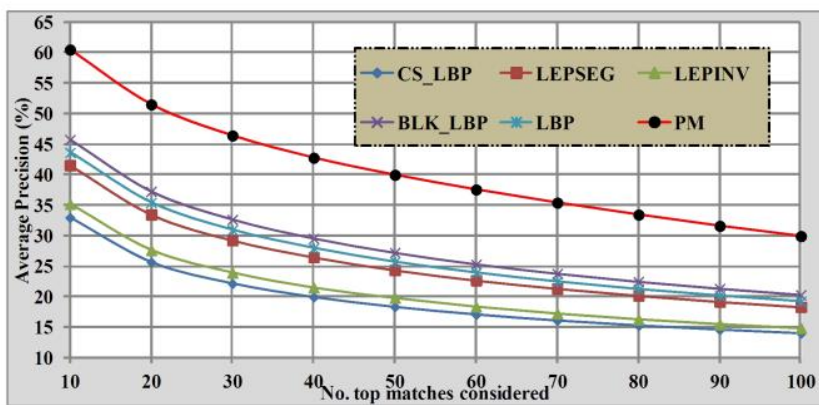

(c)

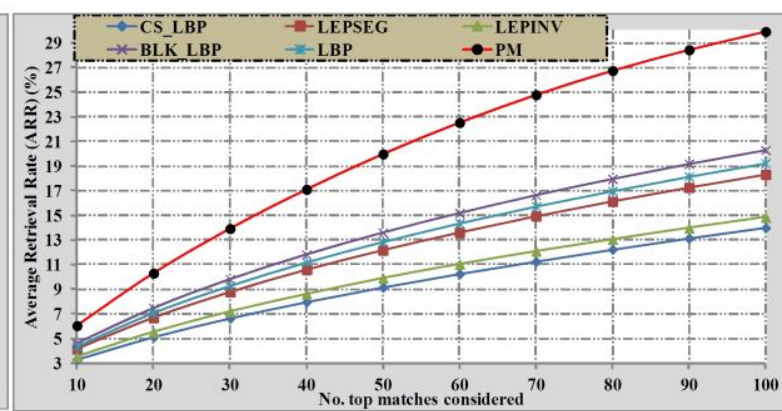

(d)

Fig. 7. Comparison of proposed method with other existing methods on Corel-5K. (a) Category wise performance in terms of precision, (b) category wise performance in terms of recall, (c) total dat abase performance in terms of average precision and (d) total database performance in terms of ARR.

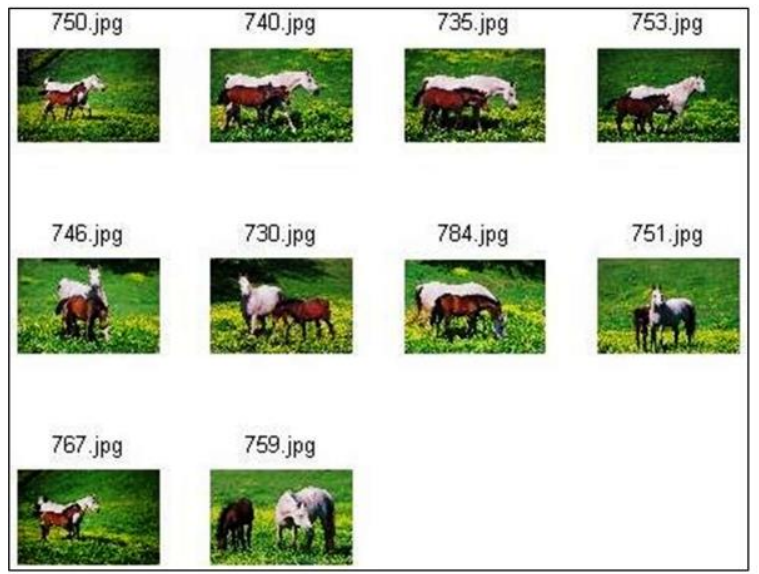

(a)

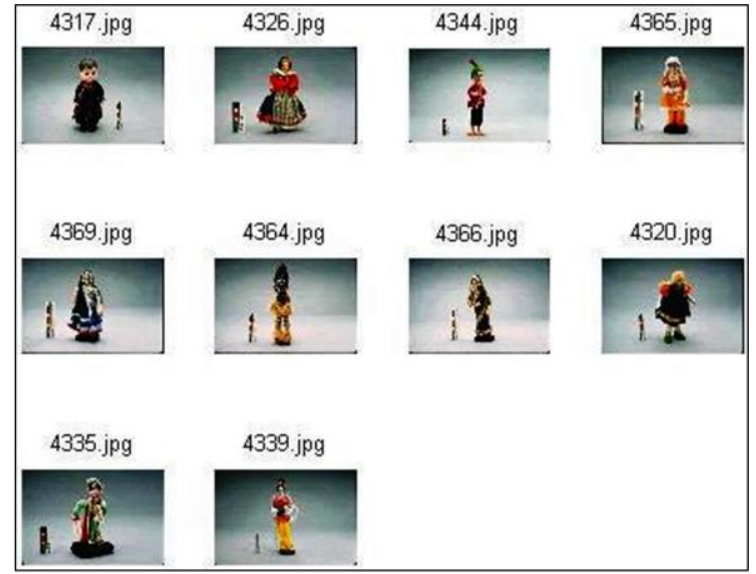

(b)

Fig. 8. Query Results of proposed method on: (a) Corel-1K and (b) Corel-5K database 


\section{CONCLUSIONS}

In this paper, a new image indexing and retrieval algorithm is proposed by combining joint histogram between first three LMEBPs, color histogram on HS spaces and LMEBP on $\mathrm{V}$ space. The experiments have been conducted on Corel- $1 \mathrm{~K}$ and Corel-5K databases for proving the worth of our algorithm. The results after being investigated show a significant improvement in terms of their evaluation measures as compared to previously available color, texture and combined color and texture features for image retrieval.

\section{REFERENCES}

[1] Y. Rui and T. S. Huang, Image retrieval: Current techniques, promising directions and open issues, J.. Vis. Commun. Image Represent., 10 (1999) 39-62.

[2] A. W.M. Smeulders, M. Worring, S. Santini, A. Gupta, and R. Jain, Content-based image retrieval at the end of the early years, IEEE Trans. Pattern Anal. Mach. Intell., 22 (12) 1349-1380, 2000.

[3] M. Kokare, B. N. Chatterji, P. K. Biswas, A survey on current content based image retrieval methods, IETE J. Res., 48 (3\&4) 261-271, 2002.

[4] Ying Liu, Dengsheng Zhang, Guojun Lu, Wei-Ying Ma, Asurvey of content-based image retrieval with high-level semantics, Elsevier J. Pattern Recognition, 40, 262-282, 2007.

[5] J. R. Smith and S. F. Chang, Automated binary texture feature sets for image retrieval, Proc. IEEE Int. Conf. Acoustics, Speech and Signal Processing, Columbia Univ., New York, (1996) 2239-2242.

[6] H. A. Moghaddam, T. T. Khajoie, A. H Rouhi and M. Saad atmand T., Wavelet Correlogram: A new approach for image indexing and retrieval, Elsevier J. Pattern Recognition, 38 (2005) 2506-2518.

[7] H. A. Moghaddam and M. Saadatmand T., Gabor wavelet Correlogram Algorithm for Image Indexing and Retrieval, 18th Int. Conf. Pattern Recognition, K.N. Toosi Univ. of Technol., Tehran, Iran, (2006) 925-928.

[8] A. Ahmadian, A. Mostafa, An Efficient Texture Classification Algorithm using Gabor wavelet, 25th Annual international conf. of the IEEE EMBS, Cancun, Mexico, (2003) 930-933.

[9] H. A. Moghaddam, T. T. Khajoie and A. H. Rouhi, A New Algorithm for Image Indexing and Retrieval Using Wavelet Correlogram, Int. Conf. Image Processing, K.N. Toosi Univ. of Technol., Tehran, Iran, 2 (2003) 497-500.

[10] M. Saadatmand T. and H. A. Moghaddam, Enhanced Wavelet Correlogram Methods for Image Indexing and Retrieval, IEEE Int. Conf. Image Processing, K.N. Toosi Univ. of Technol., Tehran, Iran, (2005) 541-544.

[11] M. Saadatmand T. and H. A. Moghaddam, A Novel Evolutionary Approach for Optimizing Content Based Image Retrieval, IEEE Trans. Systems, Man, and Cybernetics, 37 (1) (2007) 139-153.

[12] L. Birgale, M. Kokare, D. Doye, Color and Texture Features for Content Based Image Retrieval, International Conf. Computer Grafics, Image and Visualisation, Washington, DC, USA, (2006) 146 - 149.

[13] M. Subrahmany am, A. B. Gonde and R. P. Maheshwari, Color and Texture Features for Image Indexing and
Retrieval, IEEE Int. Advance Computing Conf., Patial, India, (2009) 1411-1416.

[14] Subrahmany am Murala, R. P. Maheshwari, R. Balasubramanian, A Correlogram Algorithm for Image Indexing and Retrieval Using Wavelet and Rotated Wavelet Filters, Int. J. Signal and Imaging Systems Engineering.

[15] D.G. Lowe, Distinctive Image Features from ScaleInvariant Keypoints, Int. J. Computer Vision, 60 (2) (2004) 91-110.

[16] N. Dalal and B. Triggs, Histograms of Oriented Gradients for Human Detection, IEEE Conf. Computer Vision and Pattern Recognition (CVPR), INRIA Rhone-Alps, Montbonnot, France, 2 (2005) 886-893.

[17] T. Ojala, M. Pietikainen, D. Harwood, A comparative sudy of texture measures with classification based on feature distributions, Elsevier J. Pattern Recognition, 29 (1): 51-59, 1996.

[18] T. Ojala, M. Pietikainen, T. Maenpaa, Multiresolution gray-scale and rotation invariant texture classification with local binary patterns, IEEE Trans. Pattern Anal. Mach. Intell., 24 (7): 971-987, 2002.

[19] M. Pietikainen, T. Ojala, T. Scruggs, K. W. Bowyer, C. Jin, K. Hoffman, J. Marques, M. Jacsik, W. Worek, Overview of the face recognition using feature distributions, Elsevier J. Pattern Recognition, 33 (1): 43-52, 2000.

[20] T. Ahonen, A. Hadid, M. Pietikainen, Face description with local binary patterns: Applications to face recognition, IEEE Trans. Pattern Anal. Mach. Intell., 28 (12): 2037 2041, 2006.

[21] G. Zhao, M. Pietikainen, Dynamic texture recognition using local binary patterns with an application to facial expressions, IEEE Trans. Pattern Anal. Mach. Intell., 29 (6): 915-928, 2007.

[22] M. Heikkil;a, M. Pietikainen, A texture based method for modeling the background and detecting moving objects, IEEE Trans. Pattern Anal. Mach. Intell., 28 (4): 657-662, 2006.

[23] X. Huang, S. Z. Li, Y. Wang, Shape localization based on statistical method using extended local binary patterns, Proc. Inter. Conf. Image and Graphics, 184-187, 2004.

[24] M. Heikkila, M. Pietikainen, C. Schmid, Description of interest regions with local binary patterns, Elsevie $\mathrm{J}$. Pattern recognition, 42: 425-436, 2009.

[25] M. Li, R. C. Staunton, Optimum Gabor filter design and local binary patterns for texture segmentation, Elsevie J. Pattern recognition, 29: 664-672, 2008.

[26] B. Zhang, Y. Gao, S. Zhao, J. Liu, Local derivative pattern versus local binary pattern: Face recognition with higherorder local pattern descriptor, IEEE Trans. Image Proc., 19 (2): 533-544, 2010.

[27] A. Abdullah, R. C. Veltkamp and M. A. Wiering, Fixed Partitioning and salient points with MPEG-7 cluster correlogram for image categorization, Pattern Recognition, 43, (2010) 650-662.

[28] N. Jhanwara, S. Chaudhuri, G. Seetharamanc, and B. Zavidovique, Content based image retrieval using motif co-occurrence matrix, Image and Vision Computing 22, (2004) 1211-1220.

[29] C H Lin, Chen R T, Chan Y K A., Smart content-based image retrieval system based on color and texture feature, Image and Vision Computing 27 (2009) 658-665.

[30] Subrahmanyam Murala, R. P. Maheshwari, R. Balasubramanian, "Local Maximum Edge Binary Patterns: A New Descriptor for Image Retrieval and Object Tracking," Signal Processing, vol. 92, pp. 1467-1479, 2012. 
[31] Subrahmanyam Murala, Maheshwari R.P., Balasubramanian R., Directional binary wavelet patterns for biomedical image indexing and retrieval, Proc. J. Med. Syst. doi:10.1007/s 10916-011-9764-4.

[32] Marko Heikkil, MattiPietikainen, and Cordelia Schmid, Description of interest regions with local binary patterns, Pattern Recognition, 42: 425-436, 2009.

[33] Cheng-Hao Yao, Shu-Yuan Chen, Retrieval of translated, rotated and scaled color textures, Pattern Recognition, 36: $913-929,2003$.

[34] Valtteri Takala, Timo Ahonen, and Matti Pietikainen, Block-Based Methods for Image Retrieval Using Local Binary Patterns, SCIA 2005, LNCS 3450: 882-891, 2005.

[35] Corel-1K image database. [Online]. Available: http://wang.ist.psu.edu/docs/rela-ted.shtml.

\section{Authors' Profiles}

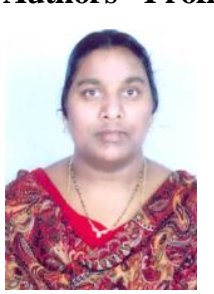

K. prasanthi Jasmine, received her B.Tech in Electronics \& Communication En gineering and M.Tech in Digital Sy stems from Regional Engineering College( Now NIT), Warangal, Andhra Pradesh, and Osmania University College of Engineering, Osmania University, Andhra Pradesh, India in the years 2000 and 2003 respectively. Currently, she is pursuing Ph.D from Andhra University, A.P, India. Her major fields of interest is Image Retrieval, Digital image Processing and Pattern Recognition.

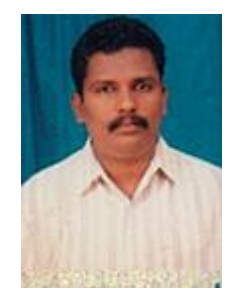

P. Rajesh Kumar received his M.Tech, $\mathrm{Ph} . \mathrm{D}$ degrees from Andhra University, Vishakhapatnam, India. $\mathrm{He}$ is currently working as professor, Department of Electronics \& Communication En gineering, Andhra University College of Engineering, Visakhapatnam, Andhra Pradesh. He is also Assistant Principal of Andhra University college of Engineering, Visakhapatnam, Andhra Pradesh. He has twenty years experience of teaching undergraduate and postgraduate students and guided a number of post graduate thesis. He has published twenty research papers in national and international journals and conferences. Presently he is guiding twelve Research scholars. His research interests are digital signal and image processing, computational intelligence, hu man computer interaction, radar signal processing.

How to cite this paper: K. Prasanthi Jasmine, P. Rajesh Kumar,"Color and Local Maximum Edge Patterns Histogram for Content Based Image Retrieval", International Journal of Intelligent Systems and Applications(IJISA), vol.6, no.11, pp.66-74, 2014. DOI: 10.5815/ijisa.2014.11.09 